\title{
Ann Arbor Pathologic Stage II
}

National Cancer Institute

\section{Source}

National Cancer Institute. Ann Arbor Pathologic Stage II. NCI Thesaurus. Code C125475.

A pathologic stage that refers to lymphoma involving two or more node regions on the same side of the diaphragm or a single extranodal organ plus its regional lymph nodes with or without other nodes on the same side of the diaphragm (IIe). 